\title{
Design and Realization of Heart Rate Signal Detection System Based on Android Mobile Phone
}

\author{
Yuxin Zhu', Jing Han ${ }^{2}$ \\ ${ }^{1}$ Institute of Information Science and Technology, Yanshan University, Qinhuangdao 066004, China \\ ${ }^{2}$ Department of Electronic Engineering, Tsinghua University, Beijing, 100084, China \\ aislinnne@163.com, \\ hanj114@163.com
}

\begin{abstract}
This paper introduces a heart rate detection system based on Android mobile phone system. The system uses Android smart mobile phone to collect the sphygmus signal from a finger, then analyzes the signal by Java program, finally gives the measured heart rate. The result shows that the system is a more accurate and more available mobile phone sphygmus detection system than the existing software in the market.

Index Terms - Android operating system; heart rate; detection
\end{abstract}

\section{Introduction}

Heart rate is an important vital sign to human. Each person's heart rate fluctuates within a normal range, any anomalous heart rate shows there is a physical health problem, so the heart rate monitoring is very essential. It can help people easily determine whether their hearts are working well, and it can also help measure the health grade of a person who is doing sports. There are many methods of monitoring the heart rate, for example, using some professional equipment, such as heart rate monitoring equipment, heart rate monitors watch or reflection type infrared heart rate detecting instrument. But these devices are not portable and they cost users a lot of money.

With the development of science and technology, mobile phone is no longer limited just to giving phone call or sending short messages. Various kinds of softwares make the smart mobile phone a more and more important role in daily life.

Because of the popularity, portability and the facile operation of smart mobile phone, using it to measure heart rate anywhere and anytime will be a promising trend in the future. There has already got a smart mobile phone heart rate detection software mentioned in reference [1], but that software is used only for Iphone which is sort of expensive for most people. Android smart mobile phone has a lower price, and the Android mobile phone system has been used in lots of companies' products for its easier twice developed platform, and strong operability ${ }^{[2-4]}$.This paper introduces a heart rate detection software which is based on Android smart mobile phone platform to meet the requirements of the times, and to bring convenience to people's lives.

\section{Main principles}

This section shows the method and implementation of image recognition and retrieval in this system.

RGB color mode uses RGB model for each pixel in the image of RGB component is assigned a $0 \sim 255$ range of intensity values. RGB images can use only three colors to mix in different proportions and reproduce 16777216 kinds of colors on the screen. The RBG color space is shown in Figure 1.

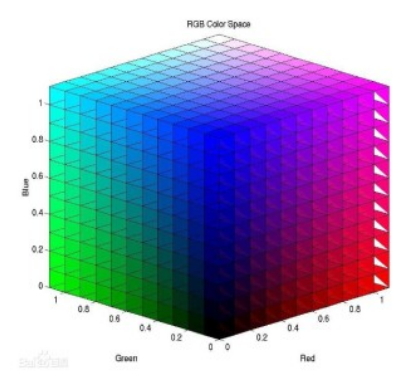

Fig. 1 RBG color space

Every pixel in a corresponding color count a RGB number, sum up these numbers in the chart on the formation of the frequency distribution of color, which is called the color histogram. There is one color histogram shown in figure 2 .
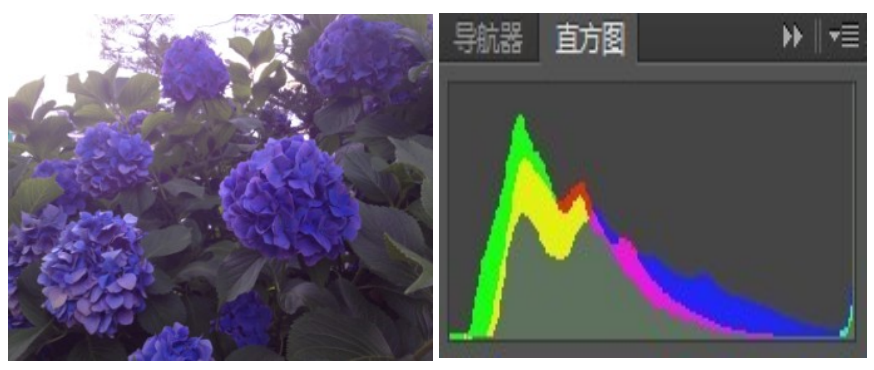

Fig. 2 Schematic diagram of color histogram

But RBG color space can't easily recognize which number represents what color, it is not convenient to code the program which is used for similarity judging. So this paper transfers RBG color space to HSV color space and uses distance function to distinguish two colors.

Human's eye perception about color mainly consists of three elements: hue $(\mathrm{H})$, saturation $(\mathrm{S})$ and value $(\mathrm{V})$, which form the HSV color space ${ }^{[5]}$. The HSV color model can be represented by an inverted cone. Long axis denotes value, leave the long distance to express saturation, and the angle around the axis to be representation of hue. HSV model is shown in Figure 3. Gray level along the axis from the bottom to the top changes from black to white. The circumference of the cone's top surface represents the maximum value and the maximum saturation of color ${ }^{[6]}$. 


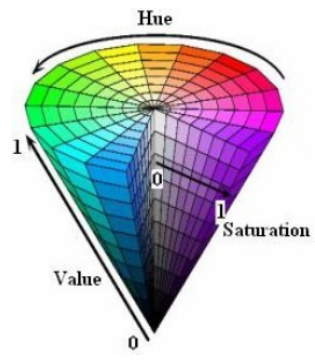

Fig. 3 HSV color space model

The process of color histogram is generally divided into five steps:

1) Partition the images and select color space;

2) Qualify the color space,

3) Calculate the distance function of the color histogram;

4) Form the color histogram after statistics;

5) Find out the most matching image by calculate similarity degree.

The system uses color histogram to analyze images. According to each part of the matching degree of color partition, using the method of similarity measure and calculating the phase distance function to retrieve the most similar image to the first frame. Besides ensuring the basic accuracy, this paper tries to make a simpler calculation process and selects the Euclidean distance as the similarity metric function.

\section{3 the Algorithm of This Paper}

\subsection{Image Partition}

In order to make the test results more accurate, this paper divides each of the images into nine equal blocks, as depicted in Figure 4.

\begin{tabular}{|l|l|l|}
\hline 1 & 2 & 3 \\
\hline 4 & 5 & 6 \\
\hline 7 & 8 & 9 \\
\hline
\end{tabular}

Fig. 4 Schematic diagram of image partition

Because the color histogram cannot distinguish the position of colors, so partition the whole graph to reduce the search range, then calculate the average index of similarity to obtain the index of similarity of the whole image, using this method to reduce the error and to improve the accuracy.

\subsection{Distance measure}

Two images set to be compared to $x_{p}=\left(x_{1}, x_{2}\right)$ and $y_{q}=\left(y_{1}, y_{2}\right)$, the Euclidean distance between them is show in (1):

$$
d\left(x_{p}, y_{q}\right)=\sqrt{\left(x_{1}-x_{2}\right)^{2}+\left(y_{1}-y_{2}\right)^{2}}
$$

\subsection{Block Cumulative Similarity}

Calculated partition of each block color histogram, similar formula between the blocks is in $(2)^{[7]}$.

$$
\operatorname{sim}\left(u_{s}, u_{t}\right)=\frac{1}{2} \sum_{i=0}^{1}\left(1-\frac{\left|x_{i}-y_{j}\right|}{\max \left(x_{i}-y_{j}\right)}\right)
$$

Now require an array to record the similarity of each block in two images, then obtain the final similarity after the addition from the following in $(3)^{[8]}$ :

$$
\operatorname{totalsim}(S, U)=\sum_{i=1}^{9} \operatorname{sim}\left[i, d_{i}(i)\right]
$$

Similar to the similarity measurement of blocks, the results is a number between 0 to 1 , closer the result to 1 , greater the similarity between images.

\subsection{Similarity Ranking}

Sequence the absolute value of image similarity, select the closest one to 1, then the frames before the selected one would be shown as a cardiac cycle.

\section{Design Program}

As the heart beats once, the blood in body has one circulation, and the vessel wall renders expansion and contraction with the heart beat. Vascular wall is thin when vascular expanses, and at this time the vascular wall has a good light transmission. Vascular wall is thick when vascular contracts, and at this time the vascular wall has a poor transparency. Fingertip is in the end of nerves, which are full of capillaries, so it is easy to see the color changes when vascular wall transmits because of the heart beat under strong light. This system uses this principle as a central point, utilizes mobile phone flashlight as a strong light source, and records a video of fingertip for several seconds. Then the system analyses the period of the color changed through the video (consider the changes from a deep color to a light color in video for a cycle) to determine the time for one heartbeat as $\mathrm{T}$, then substitutes $\mathrm{T}$ in $\mathrm{N}=60 \mathrm{~s} / \mathrm{T}$ to get $\mathrm{N}$ as the heart rate.

The system consists of four main modules which are system interface, video capture, data analysis and heart rate calculation, which are showed in Figure 5.

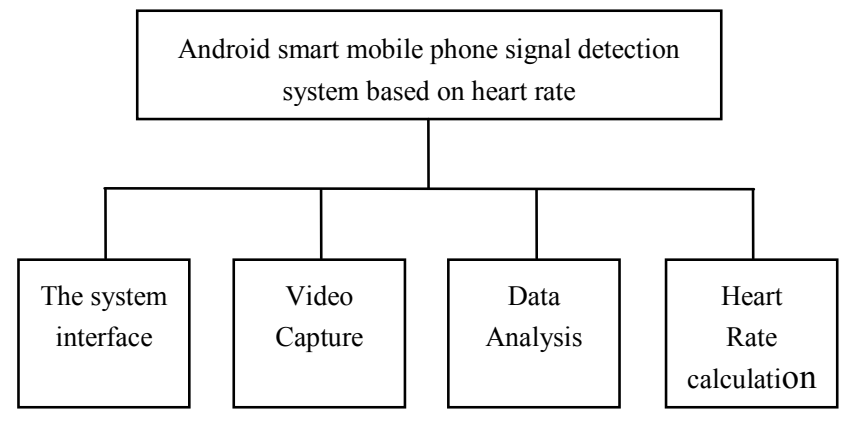

Fig. 5 modules of the measurement system

1) The interface of the system sets the start button, clicks the start button and starts measurement.

2) Call the mobile phone's rear camera to record a video of the fingertip, open mobile phone built-in flashlight as a strong light source. 
3) After recording the video, data analysis starts. Separate the video for 25 frames per second, and divide each frame of image into nine smaller equal parts, use color histogram to record colors' percentage of each of the nine small portions in the first frame image, then use color histogram to search the separated parts above, and compare with the first frame of the video. The colors' distribution percentage must be an logical trend about reaching farther at first and then reaching closer, and the search will finally find out the frame which has the most approximate percentage of the color distribution to the first frame. Record the image for the Mth frame. Substitute the data into $\mathrm{T}=\mathrm{M} / 25$, get the time $\mathrm{T}$ between the first frame and the searched frame will be a cardiac cycle.

4) Calculate the heart rate $\mathrm{N}$ for $\mathrm{N}=60 \mathrm{~s} / \mathrm{T}$.

5) The output interface can clearly shows the number of heart rate.

The flow chart of the system is shown in Figure 6.

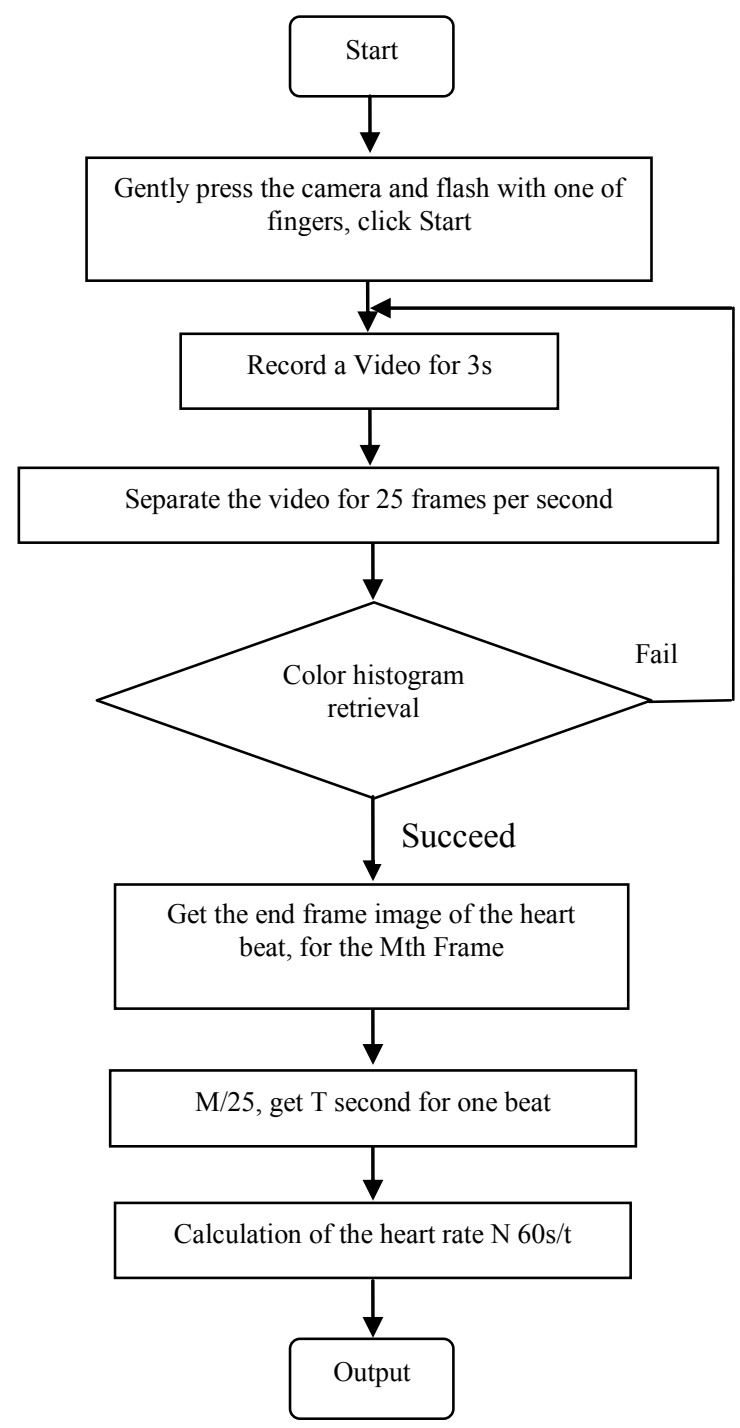

Fig. 6 The flow chart of the system

\section{Experimental results}

The system is based on Android smart mobile phone operating system and java language, using the mobile phone built-in camera for video recording. It then analyses the images using color histogram ${ }^{[9-11]}$, and simulates the system through Eclipse. This part will show some of the main principles of the system implementation method and implementation process figures.

\subsection{The System Interface}

The initial interface should firstly show "measure heart rate" as the title, and add a button to start after clicking on it. Then set the button to show "the button has been clicked" after clicking the start button. Figure $7(\mathrm{a})$ is the image of the initial interface:

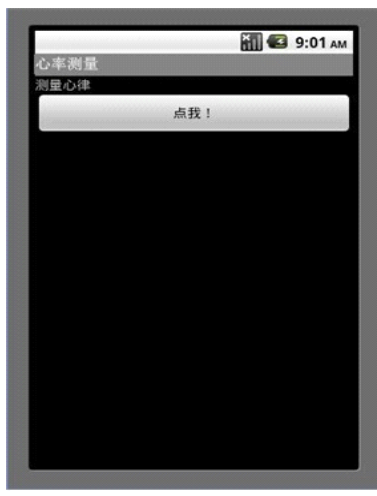

(a)

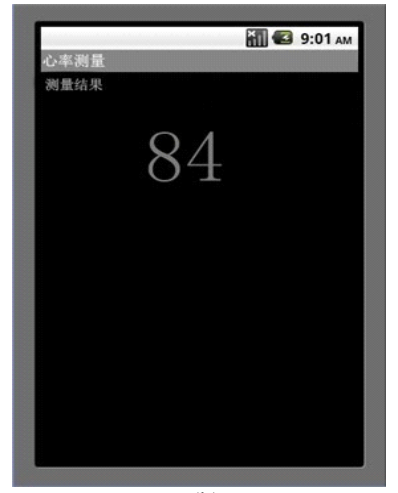

(b)
Fig.7 (a)The initial system interface;(b)System measurement result effect

After the initial interface, display the heart rate by showing the test results. The result is shown in figure 7(b).

\subsection{Video Capture}

This section will call the mobile phone's rear camera to record the video for three seconds and then let the camera implement the permanent dormancy command, use timer to control the end of this stage of the program. If recording time is too long, users will feel inconvenient, but if recording time is too short, then may not be able to catch an entire cycle. If suppose $3 \mathrm{~s}$ to be a cardiac cycle, then there will contain $60 \mathrm{~s} / 3 \mathrm{~s}=20$ cardiac cycle in $1 \mathrm{~min}$. As a normal human's heart rate cannot be less than 20 , so the video time is quite appropriate. This paper picks out six frames from the video and they are shown in the first line of figure 9.

After finishing the code in this section, the image samples for analysis are already obtained.

\subsection{Data Processing}

Decompose the video into images for 25 frames per second, divide each frame for nigh equal squares, use color histogram to analysis the corresponding part of each frame but do not output the analysis results of color histogram, find the minimum different image in color to the first frame, denote the frame as the Mth frame, then calculate the time $\mathrm{T}$ for the time of one heartbeat through $\mathrm{T}=\mathrm{M} / 25$.

In this part, the basic analysis process is in figure8: 


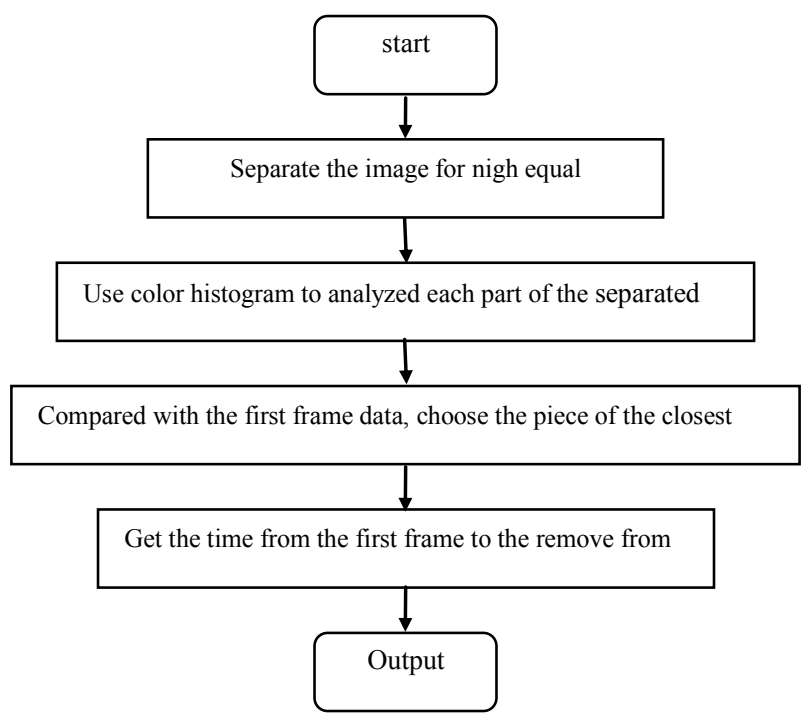

Fig.8 Data processing flow chart

Here shows six frames from the video in figure 9. The images on the first line is the frames in the video, on the second line is the HSV images of the first line, the third line is the color histograms of the Euclidean distance in HSV images on the second line.

\subsection{Get Heart Rate}

After getting the time of one heartbeat, there is only a need of one simple division, for $\mathrm{N}=60 / \mathrm{T}$, and then will obtain the final heart rate data.

\section{Conclusions}

This paper introduces the detailed process of one heart rate detection system under Android operating system. The result of the system is quite accurate through tests. With the huge development of modern society, the update of electronic products is in an increasing pace. The prospect for the mobile phone is very bright, particularly for the smart mobile phone with an Android system. So the development of appropriate applications will surely become a hot spot in the future.

\section{Acknowledgment}

This work is fully supported by Yanshan University students' extracurricular scientific research projects fund.
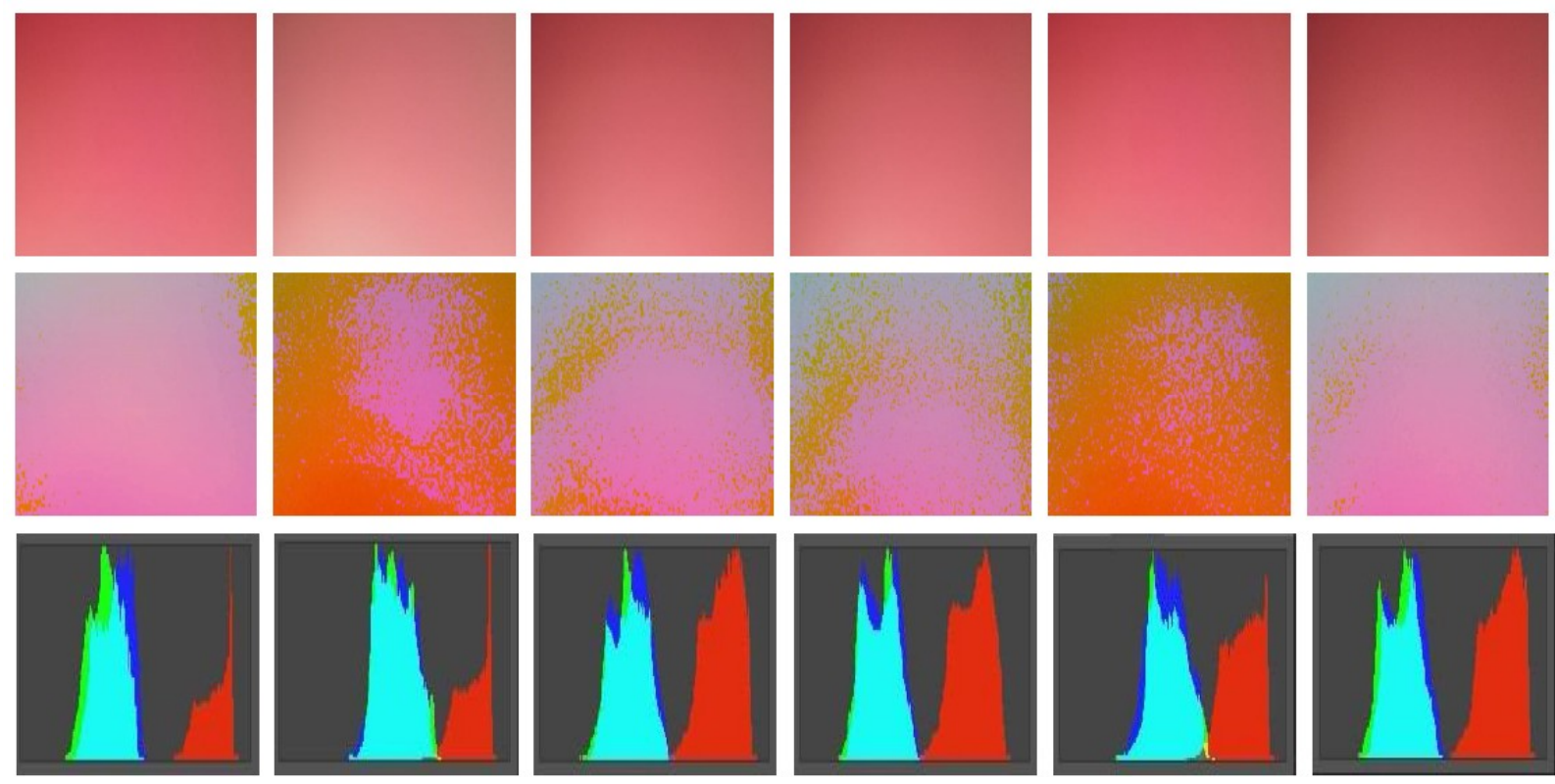

Fig.9 Sphygmus signal of a Finger and corresponding histograms

\section{References}

[1] Erika Wesley. Mobile phone can also measure heart rate [EB/OL].http://www.techreviewchina.com/story/2781.htm, 2012-08-05.

[2] Hu Wei. The Study of Android System Architecture and its Drivrs[J]. Journal of Guangzhou Open University, 2010(4):96-112.

[3] Gong Lei, Zhou Cong. Development and Research of Mobile Termination Application Based on Android[J]. Jisuanji Yu Xiandaihua,2008(8):85-89.

[4] Yao Yuwen, Liu Weiguo. Study on Android's Architecture and Its Application development[J]. Application of computer system, 2008(11):110 112.

[5] Zhang Yujin. Image Programme(Volume 1: Image processing and analysis)[M]. Beijing: Tsinghua University press, 1999.

[6] CASTLEMAN K R. Digital image processing[M]. Beijing: Electronic Industry Press, 1998
[7] Jiang Lanchi, Shen Guoqiang, Zhang Guoxuan. An image retrieval algorithem based on HSV color segment histograms[J]. Mechanical and Electrical Engineering, 2009, 26(11): 54-57

[8] Yin Ying, Two-dimensional histogram retrieval based on color space[J]. Fujian computer, 2009(10):92-93.

[9] Ying Long, Wu Lingda, Luan Xidao. Image Retrieval based on Dynamic Local Color Histogram[J]. Systems Engineering Theory and Practice, 2006(11):92-97.

[10] Yuan Guanglin, Xue Mogen, Xie kai etc. Mean Shift tracking with Multiple Color Histogram Adaptive Intergration[J]. Journal of Image and Graphics, 2011,16(10):1832-1840.

[11] Huang Jing, Ni Lin. Image Retrieval based on Radius and Angle Histograms of color Blocks[J]. Computer Engineering, 2008,34(10):202-204. 